\title{
GMC Design Based on SVM Model of Batch Reactor
}

\author{
S.Sujatha \\ Research Scholar \\ Madras Institute of Technology \\ Anna University,Chennai
}

\author{
Dr.N.Pappa \\ Assistant Professor \\ Madras Institute of Technology \\ Anna University,Chennai
}

\begin{abstract}
The use of support vector machine (SVM) in all aspects of process engineering activities, such as modeling, design, optimization and control has considerably increased in recent years. Batch reactors mostly used in chemical and pharmaceutical industries. In this paper, a design procedure of support vector machine (SVM) based model identification and control strategy for a batch reactor process with input-output form is proposed. In order to implement the generic model control structure, straight model representation and identification methods are addressed in detail. The control of a simulated batch reactor illustrates the proposed design procedure and the properties of the SVM based model identification, for nonlinear systems. This non linear control is a generic model control (GMC) is found to be well suited for a system when there is no significant rate of change of the set point.
\end{abstract}

\section{Keywords}

Support vector machine, GMC(Generic model control), batch reactor..

\section{INTRODUCTION}

Batch reactor is an essential unit operation in almost all batchprocessing industries. The control of a batch reactor is a simple case consists of charging the reactor, controlling the reactor temperature to meet the processing criterion, shutting down and emptying the reactor. Operating batch reactors efficiently and economically is very important as far as overall profitability is concerned.

The dynamic optimization (optimal control) of batch reactors has received major attention in the past. The focus of this paper is to obtain optimal operating policies of batch reactors in terms of reactor temperature and to track these temperatures by designing controllers based on non linear model. Generally, Mathematical descriptions of these complex systems are invariably difficult and hence numerical or empirical representations are usually sought for use in advanced model based control strategies[1].

Different classes of functions can be used to define empirical equations. Most of these approaches are based on the Empirical Risk Minimization principle (ERM), where the best model is found by optimizing its performance on a training data set. These model classes include multilayer perceptron and radial basis function neural networks, multivariate adaptive regression splines, projection pursuit regression, etc. The ERM principle is generally employed in the classical methods, such as least square methods, maximum likelihood methods and traditional Artificial Neural Network (ANN). A relatively new learning paradigm, SVM learning, is based on minimizing the probability of incorrectly predicting yet to be seen future outputs for a fixed, but unknown probability distribution of the data-the Structural Risk Minimization (SRM) principle. This approach has proved successful instance where other model classes have failed [2].

In SVM the ERM is replaced by the SRM principle which seeks to minimize an upper bound of the generalization error rather than to minimize the training error. Based on this principle, SVM achieves an optimum network structure by striking a right balance between the quality of the approximation of the given data and the complexity of the approximating functions. Therefore, the over fitting phenomenon in General ANN can be avoided and excellent generalization performance can be obtained. Furthermore, in SVM support vectors corresponding to the hidden units of General ANN are automatically determined after the SVM training. This implies that the difficult task of determining the network structure in general ANN can be avoided [3].

This paper is organized as follows. In section 2, model of the batch reactor is briefly presented. In section 3, SVM for nonlinear system identification of the reactor with SVM and detailed process of training and testing the SVM model are given. In section 4, design of GMC generic model control for the reactor is explained. In section 5, simulation result, conclusions and suggestions for future work are summarized.

\section{BATCH REACTOR}

A complex reaction scheme representative of many industrial reactions and used by Cott and Macchietto (1989) is used here. Reactions I and II has given in the following equation:

$$
\begin{aligned}
& A+B \rightarrow C \\
& A+C \rightarrow D
\end{aligned}
$$

Where $A, B$ are the raw materials, $C$ is the desired product and $D$ is the waste product. The model equations for the batch reactor can be written as:

$$
\begin{aligned}
& \frac{d M_{A}}{d t}=-R_{1}-R_{2} \\
& \frac{d M_{B}}{d t}=-R_{1} \\
& \frac{d M_{C}}{d t}=R_{1}-R_{2} \\
& \frac{d M_{D}}{d t}=R_{2}
\end{aligned}
$$




$$
\begin{aligned}
& R_{1}=k_{1} M_{A} M_{B} \\
& R_{1}=k_{2} M_{A} M c \\
& k_{1}=\exp \left(\frac{k_{1}^{1}-k_{1}^{2}}{(T r+273.15)}\right) \\
& k_{2}=\exp \left(\frac{k_{2}^{1}-k_{2}^{2}}{(T r+273.15)}\right) \\
& \frac{d T r}{d t}=\frac{\left(Q_{r}+Q_{j}\right)}{M_{r} C_{p r}} \\
& \frac{d T j}{d t}=\frac{\left(T_{j}^{s p}-T_{j}\right)}{\tau_{j}}-\frac{Q_{j}}{V_{j} \rho_{j} C_{p j}} \\
& Q_{r}=-\Delta H_{1} R_{1}-\Delta H_{2} R_{2} \\
& M_{r}=M_{A}+M_{B}+M_{c}+M_{D} \\
& C_{p r}=\frac{C_{p A} M_{A}+C_{p B} M_{B}+C_{p C} M_{C}+C_{p D} M_{D}}{M_{r}} \\
& Q_{j}=U A\left(T_{j}-T_{r}\right)
\end{aligned}
$$

wherein the initial values of the above mentioned process parameters of $\left[M_{A}, M_{B}, M_{C}, M_{D}, T_{j}, T_{r}\right]=[12.0,12.0,0.0,0.0$, $20.0,20.0]$ at $\mathrm{t}=0$ respectively. The reactor temperature is used as the control variable and is bounded between 20 and $100^{\circ} \mathrm{C}$ and the jacket temperature is the manipulated variable and it is bounded between 20 to $120^{\circ} \mathrm{C}$. The batch time is fixed to 120 minutes. All the nominal parameter and constant values used in the model equations are given in Table 1; more details about this model are given by Cott and Macchietto (1989).

\section{SUPPORT VECTOR MACHINE}

SVM is one of the methods whose motivation lies in maximizing the ability to generalize well from a small number of training samples by mapping the original space into a high dimensional inner product space called feature space via a kernel. SVMs solutions are characteristized by convex optimization problems [7]. So it can be applied in settling pattern recognition problems

\begin{tabular}{|c|c|c|}
\hline \multicolumn{2}{|c|}{ Constant Parameters } & Values \\
\hline$C_{p A}$ & $\begin{array}{l}\text { Specific heat capacity of } \\
\text { component A }\end{array}$ & $18.0 \mathrm{kcal} / \mathrm{kmol}^{\circ} \mathrm{C}$ \\
\hline$C_{p B}$ & $\begin{array}{l}\text { Specific heat capacity of } \\
\text { component B }\end{array}$ & $40.0 \mathrm{kcal} / \mathrm{kmol}^{\circ} \mathrm{C}$ \\
\hline$C_{p C}$ & $\begin{array}{l}\text { Specific heat capacity of } \\
\text { component } \mathrm{C}\end{array}$ & $52.0 \mathrm{kcal} / \mathrm{kmol}^{\circ} \mathrm{C}$ \\
\hline$C_{p D}$ & $\begin{array}{l}\text { Specific heat capacity of } \\
\text { component D }\end{array}$ & $80.0 \mathrm{kcal} / \mathrm{kmol}^{\circ} \mathrm{C}$ \\
\hline$\Delta H_{1}$ & Heat of reaction of reaction 1 & $-10000.0 \mathrm{kcal} / \mathrm{kmol}$ \\
\hline$\Delta H_{2}$ & Heat of reaction of reaction 2 & $-6000.0 \mathrm{kcal} / \mathrm{kmol}$ \\
\hline$C_{p}$ & Mass heat capacity of reactant & $0.45 \mathrm{kcal} / \mathrm{kg}^{\circ} \mathrm{C}$ \\
\hline$C_{p j}$ & $\begin{array}{l}\text { Molar heat capacity of } \\
\text { component } \mathrm{j}\end{array}$ & $0.45 \mathrm{kcal} / \mathrm{kg}^{\circ} \mathrm{C}$ \\
\hline$\overline{\mathrm{U}}$ & Heat transfer coefficient & $9.76 \mathrm{kcal} / \mathrm{min} m^{2}{ }^{\circ} \mathrm{C}$ \\
\hline$\rho_{j}$ & Density & $1000.0 \mathrm{~kg} / \mathrm{m}^{3}$ \\
\hline$k_{1}^{1}$ & $\begin{array}{l}\text { Pre-exponential rate constant for } \\
\text { reaction } 1\end{array}$ & 20.9057 \\
\hline$k_{1}^{2}$ & $\begin{array}{l}\text { Pre-exponential rate constant for } \\
\text { reaction } 1\end{array}$ & 10000 \\
\hline$k_{2}^{1}$ & $\begin{array}{l}\text { Pre-exponential rate constant for } \\
\text { reaction } 2\end{array}$ & 38.9057 \\
\hline$k_{2}^{2}$ & $\begin{array}{l}\text { Pre-exponential rate constant for } \\
\text { reaction } 2\end{array}$ & 17000 \\
\hline$V_{j}$ & Jacket volume & 0.6921 \\
\hline $\mathrm{A}$ & Heat transfer area & 6.24 \\
\hline $\mathrm{Mr}$ & Number of moles of component & $1560 \mathrm{~kg}$ \\
\hline$\tau_{j}$ & Jacket time constant & $3.0 \mathrm{~min}$ \\
\hline
\end{tabular}
with small samples, nonlinearity and higher dimensions. SVM can be easily introduced into learning problems such as function estimation [6].

On the basis of classical SVM, Suykens and Vandewalle [5] presented the LS-SVM approach, in which the following function is used to approximate the unknown function:

$$
y(x)=w^{T} \phi(x)+b
$$

Table 1: Nominal values of the parameters

where , $y \in R, \phi(x): R^{n}$ is a nonlinear function that maps the input space into a higher dimension feature space, $w$ 
is the weight vector and $b$ is the bias term. Then the frame work of empirical risk minimization, the cost function is formulated as

$$
\min J(w, e)=\frac{1}{2} w^{T} w+\gamma \frac{1}{2} \sum_{i=1}^{N} e_{i}^{2} ; \gamma \geq 0
$$

subject to the equality constraint

$$
y_{i}=w^{T} \phi\left(x_{i}\right)+b+e_{i}, i=1, \ldots \ldots N
$$

where $e_{i}$ is the random errors and $\gamma$ is a regularization parameter in determining the trade-off between minimizing the training errors and minimizing the model complexity. To solve this optimization problem, following Lagrange function is constructed

$$
L(w, b, e, \alpha)=\frac{1}{2}\|w\|^{2}-\sum_{i=1}^{l} \alpha_{i}\left(w^{T} \Phi\left(x_{i}\right)+b+e_{i}-y_{i}\right)
$$

where $\alpha \in \mathrm{R}$ is the Lagrange multiplier. The conditions for optimality solution can be obtained by partially differentiating with respect to $w, b, e$ and $\alpha_{i}$

$$
\begin{aligned}
& \frac{\partial L}{\partial e_{k}}=0 \rightarrow \alpha_{k}-\gamma e_{k}, k=1, \ldots N \\
& \frac{\partial L}{\partial \alpha}=0 \rightarrow w^{T} \phi\left(x_{k}\right)+b+e_{k}-y_{k}=0, k=1, \ldots N \\
& \frac{\partial L}{\partial w}=0 \rightarrow w-\sum_{k-1}^{N} \alpha_{k} \phi\left(x_{k}\right) \\
& \frac{\partial L}{\partial b}=0 \rightarrow \sum_{k-1}^{N} \alpha_{k}=0
\end{aligned}
$$

This finally result into the following SVM model for regression function (SVR)

$$
y(x)=\sum_{k-1}^{N} \alpha_{k} K\left(x, x_{k}\right)+b
$$

where $\alpha_{k}$ and $b$ are the solution to the linear system, $K(.,$.$) which is$ the kernel function satisfying the Mercer's condition represents the high dimensional feature space that is nonlinearly mapped from the input space $\mathrm{x}$. The SVR approximates the nonlinear function using equation (20). In this paper, the polynomial is used as the kernel function.

The traditional identification methods cannot build accurate mathematical models. Being different from traditional neural network, SVM is based on structural risk minimization principle, while the later on empirical risk minimization principle. In addition, because SVM algorithm is a convex optimization problem, local optimal solution must be global optimal solution. SVM utilizes optimization dual theory to make it easy to compute the estimation of model parameters in the high - dimensional feature space. The complexity of computation has little relation with the dimension or problem. SVM considers small sample enough when training, so it can solve 'over fitting' better. According to finite samples, SVM makes a good compromise between the complexity of model and learning capability to obtain best generalization[4].

In this modeling procedure, the relationship between measurable and kinetic variables of batch process can be emphasized. In order to establish the expected nonlinear model of process, we choose reactor temperature is control and input variable. The output is the desired product.

\subsection{Identification structure of SVM}

In general, a wide class of nonlinear systems can be described by nonlinear auto regressive model with exogenous inputs (NARX)

$$
\begin{aligned}
& y(k+1)=f(x(k)) \\
& x(k)=\{u(k), u(k-1), \ldots . u(k-n), \\
& y(k), y(k-1), \ldots y(k-n)\}
\end{aligned}
$$

Where $\mathrm{k}=1,2, \ldots, \mathrm{N}$.

The training sample set is first constructed and then the nonlinear sample data can be mapped as the linear outputs in high dimensional feature space by using SVM.

$$
y(k+1)=\sum_{k=1}^{N} \alpha_{k} K(x(k), x(i))+b
$$

\section{GENERIC MODEL CONTROL (GMC)}

Generic model control (GMC), a model-based control strategy developed by Lee and Suvillivan (1988) is one of the several advanced process control algorithms developed in the recent past. The GMC uses non-linear models of a process to determine the control action. The desired response can be obtained by incorporating two tuning parameters. There are several advantages that make GMC a good framework for developing reactor controllers (Cott and Macchietto, 1989):

1. The process model directly appears in the control algorithm.

2. The process model does not need to be linearised before use, allowing for the inherent nonlinearity of the exothermic batch reactor operation to be taken account.

3. By design, GMC provides feedback control of the rate of change of the control variable. This suggests that the rate of temperature change, which is very important in heat-up operations, can be used directly as a control variable.

4. The relationship between feed forward and feedback control is explicitly stated in GMC algorithm.

5. The GMC algorithm is relatively easy to implement.

It can be written as

$$
\frac{d x}{d t}=k_{1}\left(x_{s p}-x\right)+k_{2} \int\left(x_{s p}-x\right) d t
$$

Where $x$ is the current value and $x_{s p}$ is the desired value of the control variable. The first expression in the algorithm is to bring back to steady state due to change in $d x / d t$. The second expression is introduced to make the zero offset.

For temperature control of the batch reactor, a process model relating to the reactor temperature, $\mathrm{Tr}$ to the manipulated variable for the jacket temperature $\mathrm{Tj}$ is required. Assuming that the amount of heat retained in the walls of the reactor is small in 
comparison to the heat transferred in the rest of the system, an energy balance around the reactor contents gives the model in equation 8 .

Replacing $x$ with $T_{r}$ in the general algorithm the control formulation GMC is

$$
T_{j}=T_{r}+\frac{W_{r} C_{p r}}{U A}\left[k_{1}\left(T_{r s p}-T_{r}\right)+k_{2} \int\left(T_{r s p}-T_{r}\right) d t\right]-\frac{Q_{r}}{U A}
$$

The success of the GMC controller is largely dependent on the ability to measure, estimate or predict the heat release, $Q_{r}$ at any given time [8].

\section{RESULTS}

Support vector machine is used to identify the model of the batch reactor by taking the data's from the first principle model based simulated equations described in the section 2 .

With this model the GMC controller is included with $T_{j}$ as the manipulating variable and $T_{r}$ is the control variables by adjusting the tuning parameter $k_{1}, k_{2}$ to get the required desired output $M_{c}$. In figure. 3 the used values of tuning parameters are $k_{l}=0.2$ and $k_{2}$ $=1.00 \mathrm{e}-4$. These values are adjusted to get suitable output. The responses are shown in figure 1,2 and 3 .

\section{CONCLUSION}

SVM model is more attractive and it avoids using complicated differential equations to describe the process and the input output characteristics can be achieved quickly by SVM estimation. The performance of our proposed SVM modeling approach has been compared with the reference model and simulation results show that the SVM approach yield very high prediction accuracy.

With reference to the complex reaction scheme in the batch reactor the GMC controller is designed and performance are evaluated and compared with SVM model. Tuning of the GMC is required. In the future, based on this black box SVM model some optimal control scheme studies such as robust control, direct inverse model control and model predictive control can be developed.

\section{REFERENCES}

[1] M.Mujtaba,N.Aziz,M.A.Hussain, Neural network based modeling and control in batch reactor Chemical Engg Research and Design, 84(A8);635-644,2006.

[2] G.T.Jemwa,C.Aldrich, Identification of Chaotic process Systems with Least Square Support Vector Machines pp.2066 - 2071, IEEE 2003.

[3] Hai- Bo Huo, Xin- Jian Zhu, Guang- Yi Cao, Nonlinear modeling of a SOFC stack based on a least square Support Vector Machine, Journal of Power Sources 162 (2006) 1220 -1225 .

[4] Li-Na Li, Chao-Zhen Hou, The Identification of Industrial Processes based on SVM, in: proceeding of first International Conference on Machine Learning and Cybernetics, Beijing, 4-5 Nov 2002.

[5] Suykens JAK, Vandewalle J. Least squares support vector machine classifiers.Neural Process Lett, 9(3):293-300, 1999.

[6] Liu B, Su H, Huang W, Chu J. Temperature predictive control based Least Squares Support Vector Machines.J Contr Theory 4;4:365-70, April 2000.

[7] Liling Ma, Junzheng Wang, zhigang Liu, A Fault Diagnosis method based on composite model and SVM for fermentation process ,IEEE International conference on Control and Automation 2007.

[8] Lee.P.L., and Suvillivan,G.R.,Generic model control, Comput Chem Eng, 12:573,1988

[9] Cott, B.J, Macchietto, S.Temperature Control of Exothermic Batch reactors using Generic Model Control, Industrial Engineering Chemical Research.28,1177-1184,1989

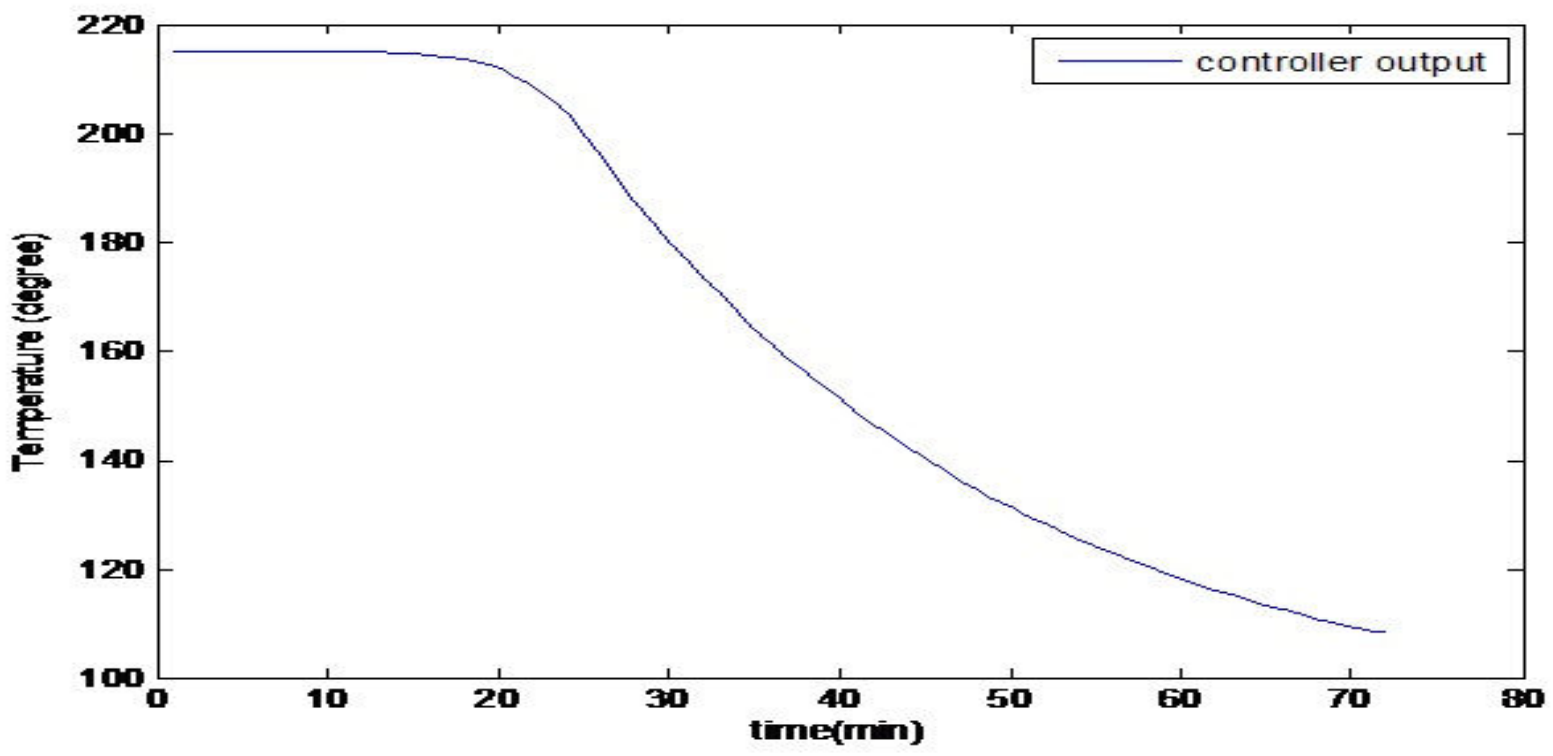

Figure 1. Reactor temperature as control variable 


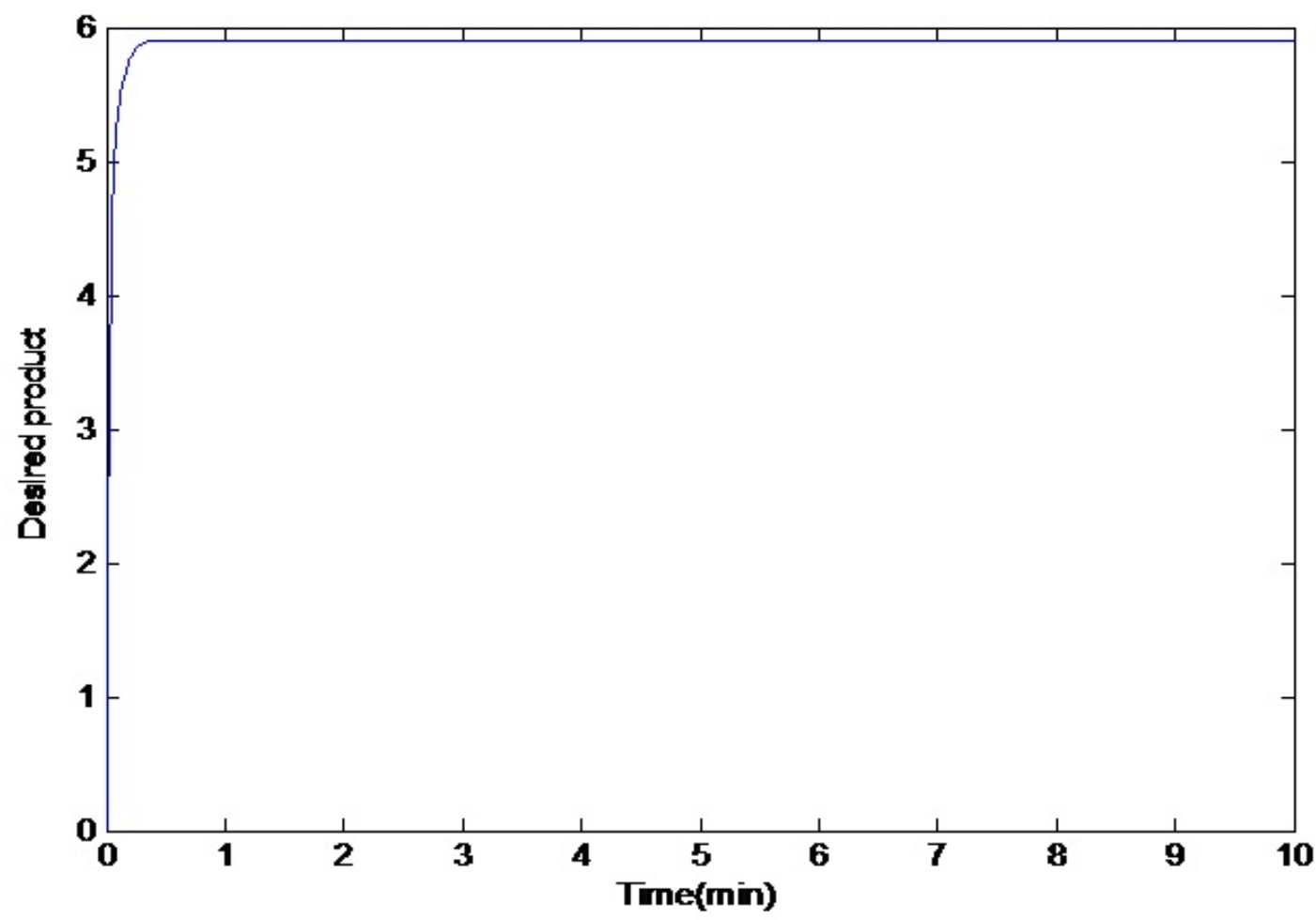

Figure 2. Output Response (Desired product).

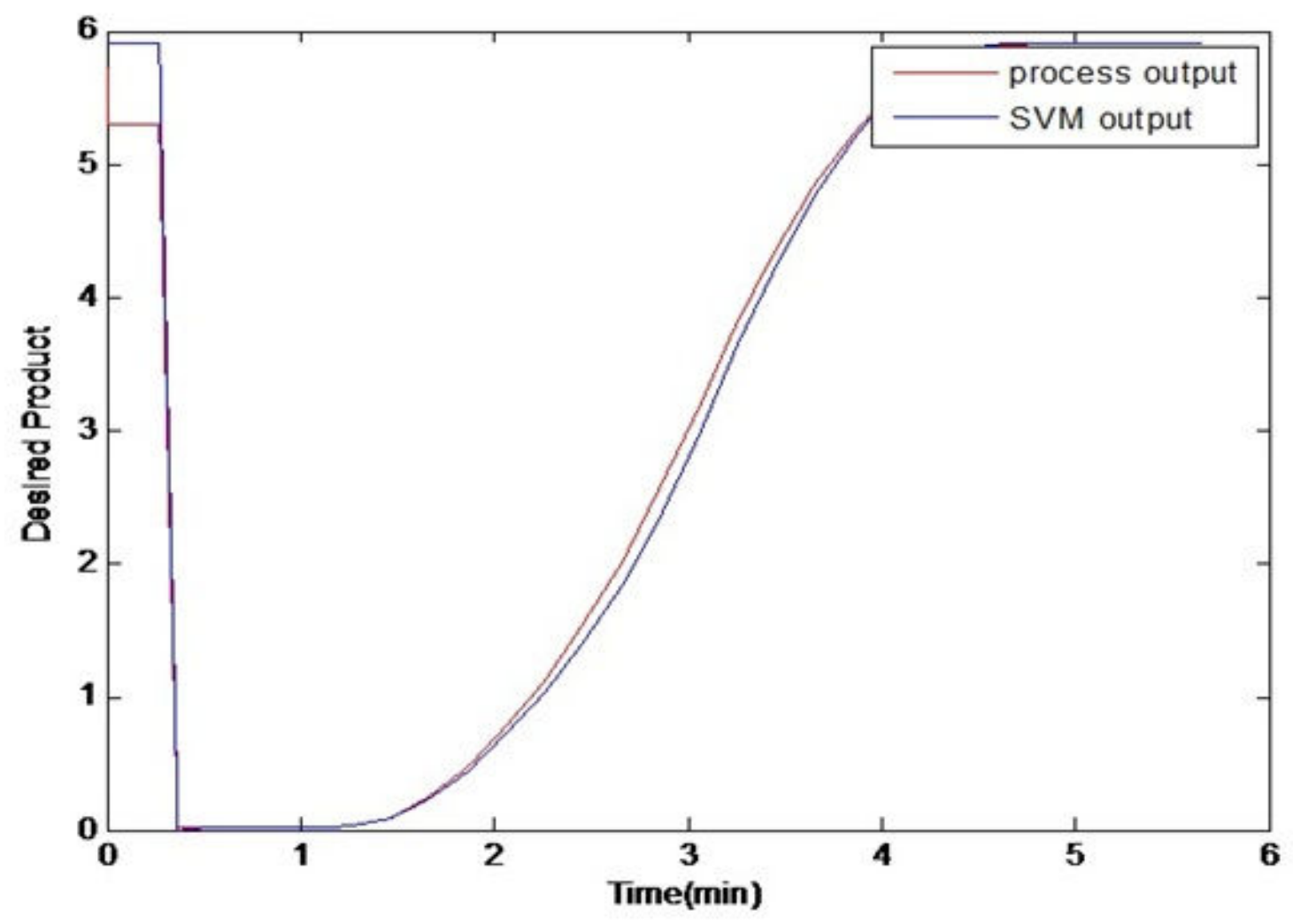

Figure 3.GMC response for dynamic setpoint. 